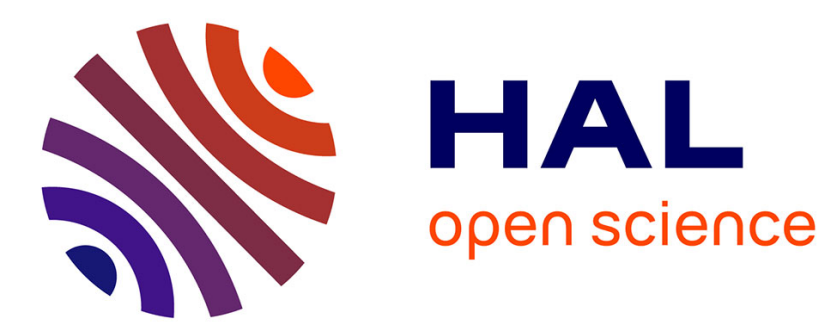

\title{
Biomimetic Approach for Highly Selective Artificial Water Channels Based on Tubular Pillar[5]arene Dimers
}

Dmytro Strilets, Shixin Fa, Arthur Hardiagon, Marc Baaden, Tomoki Ogoshi, Mihail Barboiu

\section{- To cite this version:}

Dmytro Strilets, Shixin Fa, Arthur Hardiagon, Marc Baaden, Tomoki Ogoshi, et al.. Biomimetic Approach for Highly Selective Artificial Water Channels Based on Tubular Pillar[5]arene Dimers. Angewandte Chemie International Edition, 2020, 59 (51), pp.23213-23219. 10.1002/anie.202009219 . hal-03028876

\section{HAL Id: hal-03028876 https://hal.science/hal-03028876}

Submitted on 27 Nov 2020

HAL is a multi-disciplinary open access archive for the deposit and dissemination of scientific research documents, whether they are published or not. The documents may come from teaching and research institutions in France or abroad, or from public or private research centers.
L'archive ouverte pluridisciplinaire HAL, est destinée au dépôt et à la diffusion de documents scientifiques de niveau recherche, publiés ou non, émanant des établissements d'enseignement et de recherche français ou étrangers, des laboratoires publics ou privés. 
How to cite:

\title{
Biomimetic Approach for Highly Selective Artificial Water Channels Based on Tubular Pillar[5]arene Dimers
}

\author{
Dmytro Strilets, Shixin Fa, Arthur Hardiagon, Marc Baaden, Tomoki Ogoshi, * and \\ Mihail Barboiu*
}

\begin{abstract}
Artificial water channels mimicking natural aquaporins (AQPs) can be used for selective and fast transport of water. Here, we quantify the transport performances of peralkyl-carboxylate-pillar[5]arenes dimers in bilayer membranes. They can transport $\approx 10^{7}$ water molecules/channel/ second, within one order of magnitude of the transport rates of AQPs, rejecting $\mathrm{Na}^{+}$and $\mathrm{K}^{+}$cations. The dimers have a tubular structure, superposing pillar[5]arene pores of $5 \AA$ diameter with twisted carboxy-phenyl pores of $2.8 \AA$ diameter. This biomimetic platform, with variable pore dimensions within the same structure, offers size restriction reminiscent of natural proteins. It allows water molecules to selectively transit and prevents bigger hydrated cations from passing through the $2.8 \AA$ pore. Molecular simulations prove that dimeric or multimeric honeycomb aggregates are stable in the membrane and form water pathways through the bilayer. Over time, a significant shift of the upper vs. lower layer occurs initiating new unexpected water permeation events through toroidal pores. $\square$ Abstract was shortened to fit within allotted space. Please check.
\end{abstract}

$\mathbf{N}$ atural channels allow ion/water translocation across biological membranes. ${ }^{[1]}$ The transport is regulated by proteins, converting the ion-water interactions into ion-selectivity filter ones. ${ }^{[2]}$ Because of the high significance of the related

[*] D. Strilets, Dr. M. Barboiu

Institut Europeen des Membranes, Adaptive Supramolecular Nanosystems Group, University of Montpellier, ENSCM-CNRS

Place E. Bataillon CC047, 34095 Montpellier (France)

E-mail: mihail-dumitru.barboiu@umontpellier.fr

Dr. S. Fa, Prof. T. Ogoshi

Department of Synthetic Chemistry and Biological Chemistry

Graduate School of Engineering, Kyoto University

Katsura, Nishikyo-ku, Kyoto 615-8510 (Japan)

E-mail:ogoshi@sbchem.kyoto-u.ac.jp

Prof. T. Ogoshi

WPI Nano Life Science Institute, Kanazawa University

Kakuma-machi, Kanazawa, 920-1192 (Japan) (Japan)

A. Hardiagon, Dr. M. Baaden

CNRS, Université de Paris, UPR 9080, Laboratoire de Biochimie Théorique

13 rue Pierre et Marie Curie, F-75005, Paris (France)

A. Hardiagon, Dr. M. Baaden

Institut de Biologie Physico-Chimique-Fondation Edmond de Rotschild, PSL Research University

Paris (France)

(2) Supporting information and the ORCID identification number(s) for the author(s) of this article can be found under: https://doi.org/10.1002/anie.202009219. processes, the design of synthetic unimolecular or selfassembled channels with water-ion ${ }^{[3]}$ or ion-ion ${ }^{[4,5]}$ selectivity have become areas of expanding interest. Very attractive strategies have been developed based on the self-assembly, towards supramolecular capsules presenting conductance states in bilayer membranes. Barboiu et al. ${ }^{[6]}$ Fyles et al. ${ }^{[7]}$ Kim et al. ${ }^{\left[{ }^{[8]}\right.}$ Gokel et al. ${ }^{[9]}$ and Furkawa et al. ${ }^{[10]}$ demonstrated the efficiency of integrated capsules as selectivity ion filters across lipid bilayers.

The easily accessible Pillar[ $n]$ arenes-PA play an important role in supramolecular chemistry, towards the generation of light-harvesting or drug delivery systems, transmembrane channels, separation and storage materials, etc. ${ }^{[11,12]}$ Hou et al. firstly realized that PAs, with their unique hollow pillar-shape and tuneable size cavities with fine-tuneable rims, could be suitable platforms to construct artificial channels. ${ }^{[13-24]}$ Most of them, are positioning the PA scaffolding relay in the middle of the membrane, whereas functional groups attached to aromatic rings form unimolecular pillared channels. Significant progress has been obtained in a very short time, as the singlechannel osmotic water permeability for peptide-PAPs, ${ }^{[2,26]}$ is close to natural aquaporins and superior to others synthetic water channel analogues. ${ }^{[27]}$ Their drawback it relates to their low ion-water selectivity.

Innovative design approaches for the construction of PAboxes ${ }^{[28]}$ or PA-tubular dimers ${ }^{[29]}$ were proposed. We recently described the dimerization of rim-differentiated pillar[5] arene via hydrogen bonding (Figure 1), producing expanded length-controlled tubular superstructures, ${ }^{[29]}$ reminiscent to calixarene ${ }^{[30]}$ capsules.

Pursuing our endeavors to design original supramolecular channels with efficient and selective transport properties, we recently got interested in the possibility to make use of a directional structuring of such tubular PA platforms, towards the generation of directional channels within lipid bilayer membranes. We anticipated they are able to form supramolecular self-assembled pores inside lipid bilayer membranes. As observed for alkyl-pyrogallol-arenes, the pore formation is strongly depending on the nature of grafted alkyl chains and probably more than on type of aggregates form efficient ion channels. ${ }^{[9]}$

We know that a pore with a diameter of $\approx 3 \AA$ is a critical prerequisite to selectively accommodate and transport waterwires. ${ }^{[27]}$ Pillar[5]arene platform presents a hydrophobic cavity of $5 \AA$ diameter, which in turn is much bigger to selectively accommodate single water-wires. ${ }^{[12,29]}$ Having an inner pore size of $\approx 5 \AA$, they allow the passage of cations according to their hydration energy. ${ }^{[23-25]}$ 


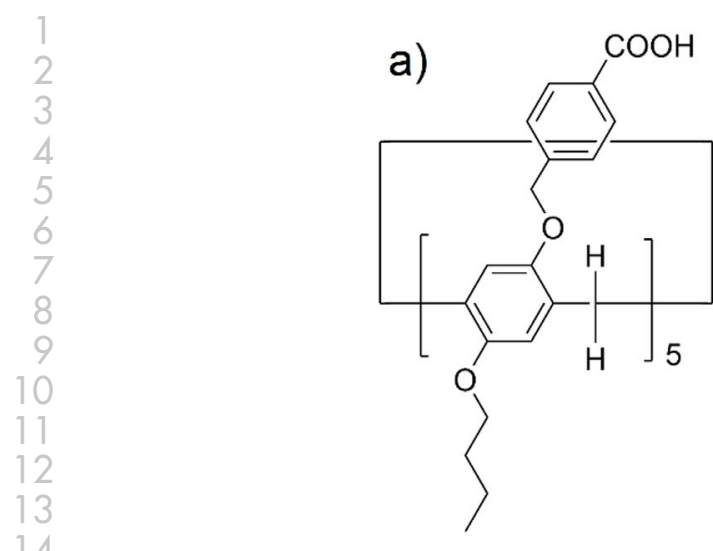

C4

b)
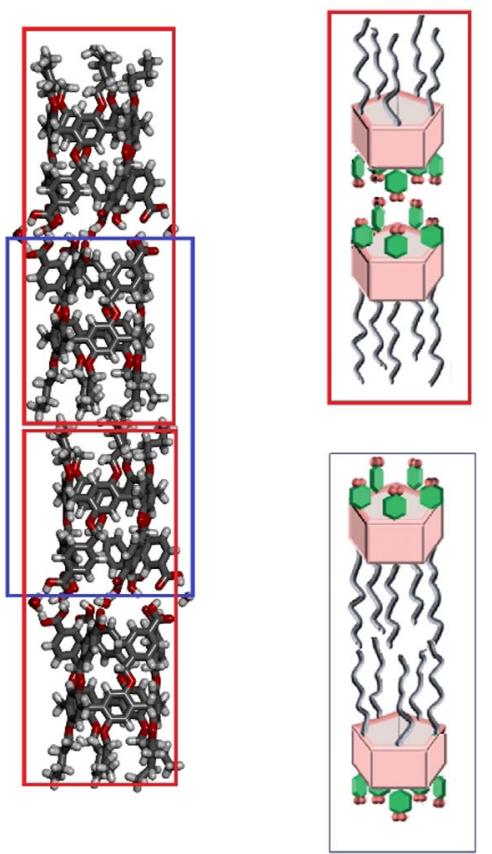
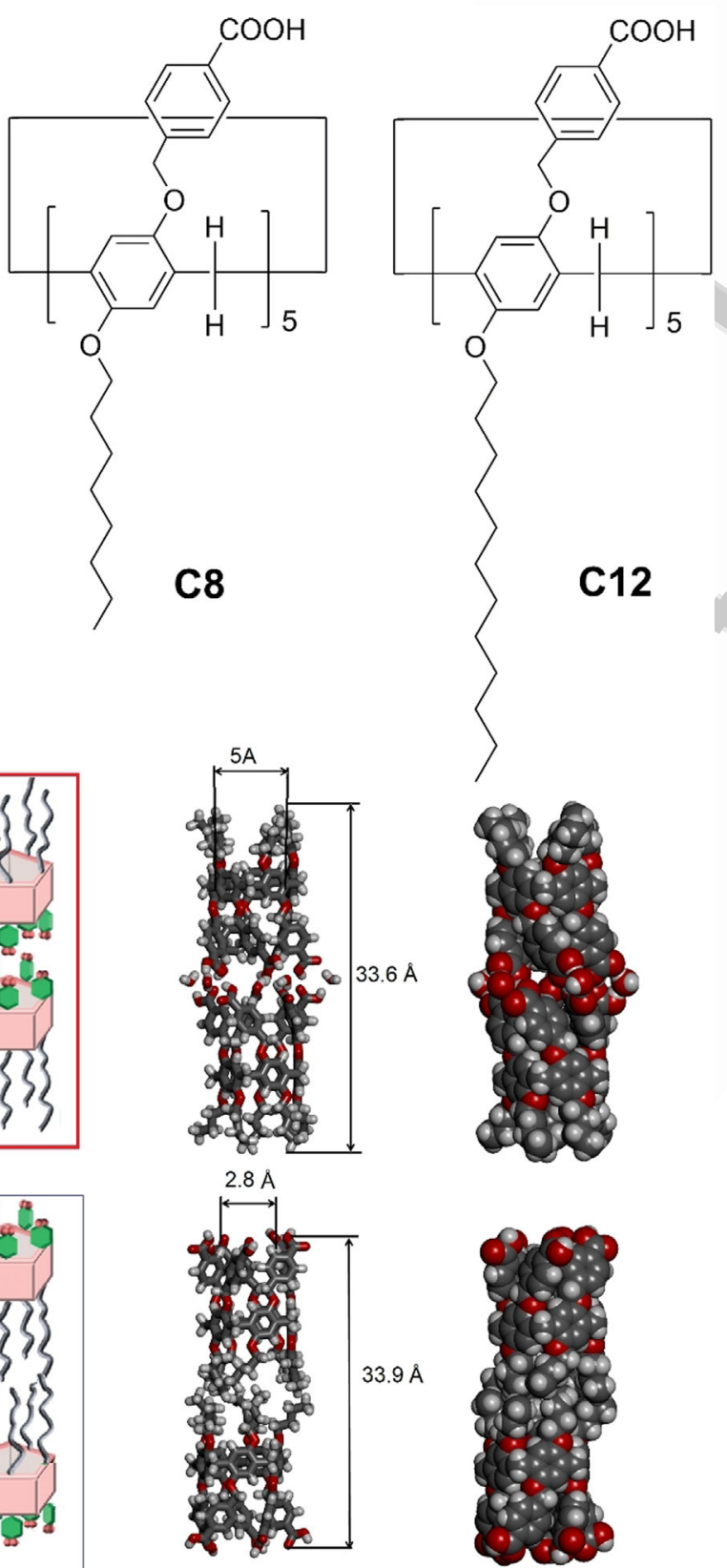

c)
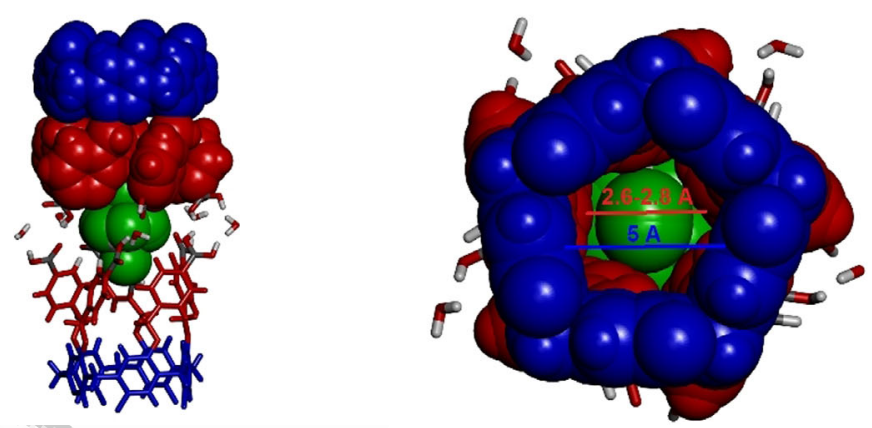

Figure 1. a) Structures of peralkylcarboxylate-pillar[5]arenes PAD4, PAD8 and PAD12 and b) X-ray single crystal structure of PAD4. ${ }^{[29]}$ Crystal packing along $c$-axis emphasizing self-assembled a) tubular carboxylate $\mathrm{H}$-bonding (red) and b) bilayer hydrophobic (blue) dimers in stick and CPK representations Crystalline solid state structures show dimeric structures of $34 \AA$ length and c) a variable pore geometry composed of Pillar[5]arene platform (blue) of $5 \AA$ diameter and of narrowest twisted carboxy-phenyl pore of $2.8 \AA$ diameter (red). $\mathrm{Green}_{\mathrm{CHCl}}$ molecules in $\mathrm{CPK}$ representation are perfectly positioning a $\mathrm{Cl}$ atom fitting the narrowest carboxy-phenyl pore, while the other atoms are disordered in the larger junction formed via hydrogen bonding of the carboxylate groups. 
Herein we concentrate our efforts on investigation of water/ion transport abilities of self-assembled discrete tubular dimers of rim-differentiated peralkyl-carboxylate-pillar[5]arenes PAD4, PAD8 and PAD12 (Figure 1a) through bilayer lipid membrane. Interestingly, the analysis of $x$-ray single structure of PAD4 show that two dimeric tubular architectures. It results in the formation of tubular carboxylic dimers $\mathrm{pS}-\mathrm{pS}$ and $\mathrm{pR}-\mathrm{pR}$ and of bilayer dimers form via two different H-bonding vs. hydrophobic binding modes, respectively (Figure $1 \mathrm{~b}$, red). The 15 hydrogen bonds between the carboxyl groups and water molecules and adopts closely spaced carboxy-phenyl groups with a fivefold helical conformation, ${ }^{[29]}$ which in turn superpose the pillar[5]arene relay of $5 \AA$ diameter with a narrowest twisted hydrophobic pore of $2.8 \AA$ diameter (Figure 1c). This affords a size restricted control of translocation along tubular channels at narrowest selectivity filter level, offering size restriction. The biological $\mathrm{KscA}^{[2 \mathrm{a}]}$ or Aquaporin ${ }^{[2 \mathrm{~b}]}$ channels, have hydrophobic conical pores with variable diameter with the selectivity filter in the middle of the channel, affording closely spaced binding sites for temporarily coordinating the partly dehydrated cations or water molecules, respectively. We anticipated, PAD4, PAD8 and PAD12 dimers, with a variable pore diameter would allow to control of the transport activity as for natural proteins, within the selectivity filter. A reverse bilayer dimer of PADs may form (Figure 1b, red), allowing phospholipid polar headgroups of the membrane to be in contact with the carboxylic rims in the polar region of the bilayer and of the butyl chains hydrophobic interactions inside the bilayer (Figure 1b, blue).

Longer alkyl chains in PAD12 might stabilize the bilayer conformation, while shorter alkyl chains in PAD4 should strongly stabilize the tubular dimers in the bilayer. It is important to note that the thickness of the insulating bilayer membrane $(30-35 \AA)^{[1]}$ is quite similar to the length of the dimeric PAD4 (Figure 1), while the hydrophobic interactions between the longer alkyl chains in PAD8 and PAD12 and the bilayer stabilize their positionings.

PAD4, PAD8 and PAD12 assemble into functional waterpermeable channels in liposomes and increase the overall water permeability relative to the background lipid permeability. ${ }^{[31]}$ Their water transport rates are strongly dependent on the nature of hydrophobic alkyl tails grafted onto pillar[5] arene platform. PAD4, PAD8 and PAD12 channels were reconstituted into phosphatidylcholine (PC) lipid vesicles (100 $\mathrm{nm}$ in diameter) with different molar ratios of compound to lipids $(0.33 \%, 0.66 \%, 1.33 \%, 2.26 \%$ and $2.99 \% \mathrm{mCLRs})$ in which channels were added by simple addition of the dimethylsulfoxide (DMSO) solutions of the PADs derivatives to pre-formed vesicle suspensions. Then the vesicles were exposed to outwardly directed osmotic pressure gradients (shrinking mode of the vesicles). Under hypertonic conditions driven by outwardly $10 \mathrm{mM}$ HEPES buffer solution 100 , $200 \mathrm{mM} \mathrm{KCl}$ osmolate, the shrinkage of the liposomes under osmotic gradients of 100,200 mOsm increased the lightscattering signal (Figure $2 \mathrm{a}$ ). The net permeabilities increase when mCLRs ratios increase and with increasing osmolarity. The permeabilities present a maximum of $9.00 \mu \mathrm{m} \mathrm{s}^{-1}$ at high $\mathrm{mCLR}=3.00$ for PAD4 which is the most permeable toward water in the dimeric tubular configuration in the series, whereas for PAD8, and PAD12, the maxima values are lower: $3.66 \mu \mathrm{m} \mathrm{s}^{-1}$ and $2.23 \mu \mathrm{ms}^{-1}$, respectively and they can be detected at $\mathrm{mCLR}=1.33 \%$ (Figure $2 \mathrm{~b}-\mathrm{d}$ ). We further estimated the single-channel permeability, $\mathrm{P}_{s}$ of each channel ${ }^{[27]}$ (see Supporting information for details). In the shrinking mode, PAD4, PAD8 and PAD12 show a tendency of $\mathrm{P}_{s}$ increasing with osmolarity as it was with net permeability. For example permeabilities of $1.54 \times 10^{7}, 5.21 \times 10^{7}$ and $7.95 \times$ $10^{6}$ water moleculess $\mathrm{s}^{-1}$ channel, are obtained for PAD4 $(\mathrm{mCLR}=1.33), \quad$ PAD8, $\quad(\mathrm{mCLR}=0.33) \quad$ and $\quad$ PAD12 $(\mathrm{mCLR}=0.33)$ respectively, which are only one orders of magnitude lower than that of AQPs $\left(\approx 10^{8}-\right.$ $10^{9}$ water molecules $\mathrm{s}^{-1} /$ channel). ${ }^{[2 \mathrm{~b}]}$ It can be noticed that with increasing of mCLR the single channel permeability is decreasing, but remains on the same level of magnitude. Thus, the tendency for dropping of single channel permeability can be explained by progressive higher aggregation or lower solubility of PAD channels at high concentration, while using low concentration for incorporation into lipid bilayer prevents its aggregation. Water transport activities decrease substantially as the grafted alkyl chain length increases (PAD4 $\gg$ PAD8 $>$ PAD12), implying the formation variable architectures of permeable tubular dimers for PAD4 and less permeable bilayer configuration for PAD12, whereas PAD8 present an intermediate situation. An unfavorable hindering wrapping of the hydrophobic tails inside the selective pore of the PADs or around at the interface with the bilayers, can be presumed for longer chains. Indeed, the tail length seemed to be optimal for butyl chains while longer chains led to aggregation when mixed with lipids.

In order to have more precise structural support for the inmembrane contentions we further prepared the carboxylate $\mathrm{Na}^{+}$or $\mathrm{Li}^{+}$salts of PAD4. ${ }^{[32]}$ Oppositely to carboxyl functionalized PAD4, for the carboxylate salt of PAD4 the hydrogenbonding driven dimerization would not be possible. PAD4Na or PADLi salts could dimerize only through the interdigitation of the alkyl chains, having the carboxylate groups interacting with the polar heads of the phospholipids. Their net and single channel permeabilities at $1.33 \%$ mCLRs are $13.00 \mu \mathrm{m} \mathrm{s}^{-1}$ and $2.83 \times 10^{7}$ water molecules s ${ }^{-1} /$ channel, re- $^{-}$ spectively, which is almost double to that observed for PAD4 counterpart, providing clear evidence two distinguish both models. For the carboxylate appended bilayer dimers the average fraction of channels filled with water is increasing due the presence of hydrophilic carboxylate groups, remaining accessible to water from both sides of the membrane.

Ion $\left(\mathrm{Na}^{+}\right.$and $\left.\mathrm{K}^{+}\right)$transport activities across the bilayer membranes incorporating PAD4, PAD8 and PAD12 channels reconstituted into phosphatidylcholine (PC) lipid vesicles $(100 \mathrm{~nm})$ at the same mCRLs as used for water transport, were assessed using standard HPTS fluorescence assays. ${ }^{[33]}$ Indeed, when tested with $\mathrm{NaCl}$ or $\mathrm{KCl}$ on external buffer, the PAD4, PAD8 and PAD12 channels had an unexpected behaviour: after the compound injection at $t=20 \mathrm{~s}$, the internal $\mathrm{pH}$ remains stable and only the creation of a $\mathrm{pH}$ gradient, applied at $t=40 \mathrm{~s}$, induce an abrupt increase of the internal $\mathrm{pH}$, reminiscent with a low $\mathrm{H}^{+}$efflux $\left(A_{\max }=0.06\right.$ for PAD4, 0.10 for PAD8 and 0.03 for PAD12 at $300 \mathrm{~s}$ ) (Figure $\mathrm{S} 7$ ) and then the internal $\mathrm{pH}$ remains quasi-stable, 
a)
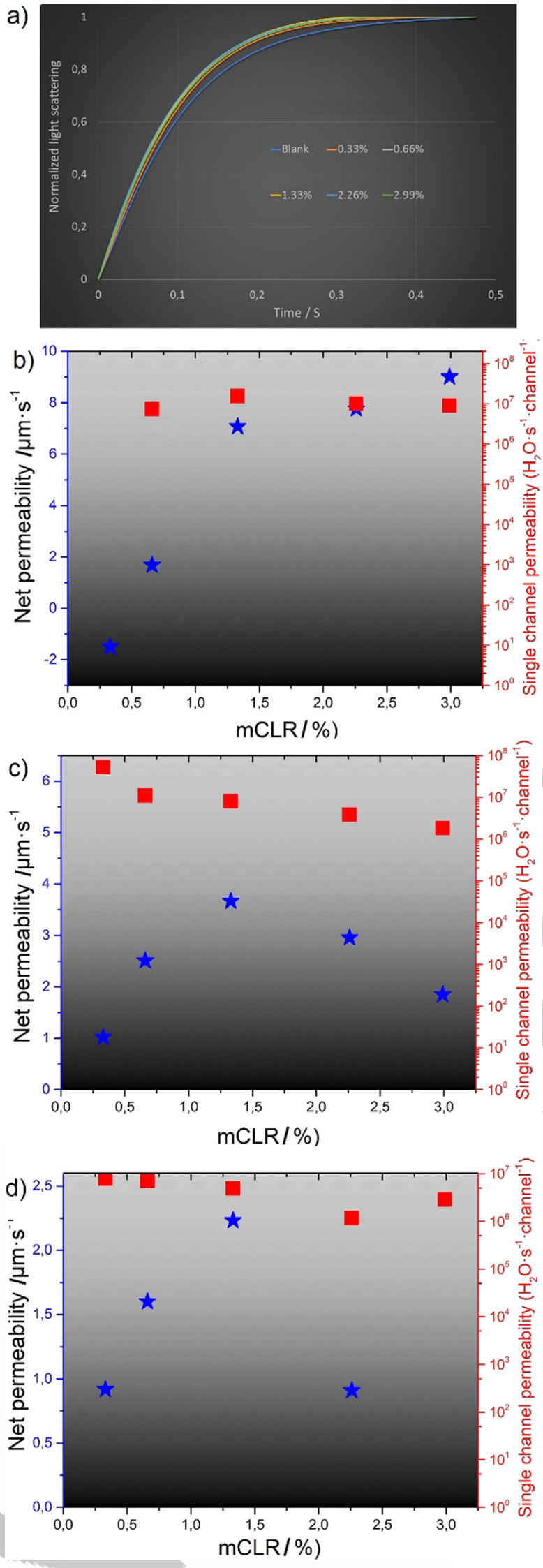

Figure 2. Water-transport activity in liposomes: a) Representative stopped-flow light-scattering experimental traces of liposome post-mix assays at different mCLRs of PAD4 after abrupt exposure to a hypertonic solution of $200-\mathrm{mM} \mathrm{KCl}$. The net and single water permeabilities of the liposomes containing b) PAD4, c) PAD8 and d) PAD12 channels at different $\mathrm{mCLRs}$, measured under hypertonic conditions abruptly exposed to a hypertonic solution of $100-\mathrm{mM} \mathrm{KCl}$ from the post-mix assays.

independent on the nature of the channel or transported cation. Accordingly, the addition of base in the extravesicular solution induces a rapid deprotonation of carboxyl groups of the channel in the bilayer membrane, which synergistically induce HPTS deprotonation and generate the observed abrupt increase in fluorescence. After $\mathrm{pH}$ equilibration, dose-response experiments show the constant absorbance behaviors. It is noteworthy that, $\mathrm{K}^{+}$and $\mathrm{Na}^{+}$cations are not transported and channel structuration is no effective to induce $\mathrm{Na}^{+}$or $\mathrm{K}^{+} / \mathrm{H}^{+}$antiport conductance states along the assembled channel.

Single channel planar lipid bilayer experiments confirm the complete inactivity for $\mathrm{K}^{+}$-transport under applied potential in range of $50-200 \mathrm{mV}$ for tested solutions of PAD4 (Figure S8). The transport activity is rather impossible to initiate, both in terms of length of operating periods and the concentration of PAD4 in the membrane.

Overall, the PAD4, PAD8 and PAD12 dimers, obtained using simple chemistry, can be assembled into highly selective artificial water channels and remain stable in lipid membranes. Previously, the seminal discovery of pillar[5]arene PAP unimolecular channels by $\mathrm{Hou}$ et al. ${ }^{[15]}$ are at long last beginning to meet encouraging signs for high water permeability $\left(10^{6}-10^{8}\right.$ water molecules/second/channel), while most of these channels present ionic transport activity, thus not yet selectivity. Natural AQP channels present an hourglass structure offering size $\mathrm{SF}$ restriction of $\approx 3 \AA$ and selectivity against cations, reinforced through electrostatic repulsion in the region known as the aromatic arginine $(\mathrm{ar} / \mathrm{R})$ constriction. ${ }^{[2 \mathrm{~b}]}$ In this study a simple serendipitous modification of rims of pillar[5]arene backbone of $5 \AA$ diameter led to the construction of a supplementary narrowest hydrophobic pore of 2.6-2.8 $\AA$ diameter within the same structure, affording a size restriction control of translocation along tubular dimers at this selectivity filter level. The water permeability of PAD4, PAD 8 and PAD12 channels $\left(\approx 10^{7}\right.$ water molecules $\mathrm{s}^{-1} /$ chan- $^{-}$ nel) is only one orders of magnitude lower than that of AQPs, and more important they are rejecting alkali cations. Differently to previous selective I-quartets where water molecules are interacting along the whole structure of the channel of $\approx 2.8 \AA$, herein the narrowest pore structure of PAD4, PAD8 and PAD12 channels in precisely located at the two narrowest regions of the pore, which remains larger along the resting length of the pore. The narrowest diameter of the pore itself is large enough for the water molecules to pass having themselves a diameter of $\approx 2.8 \AA$, but restrictive enough to block the passage of hydrated $\mathrm{Na}^{+}$or $\mathrm{K}^{+}$cations.

We set up computer simulations to probe the behavior of PAD4 dimers in an explicit lipid bilayer environment. Simulation results are summarized in Figure 3. As a first test 
a)

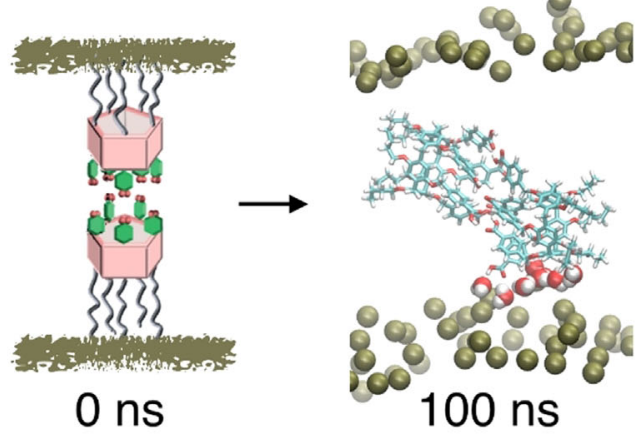

b)

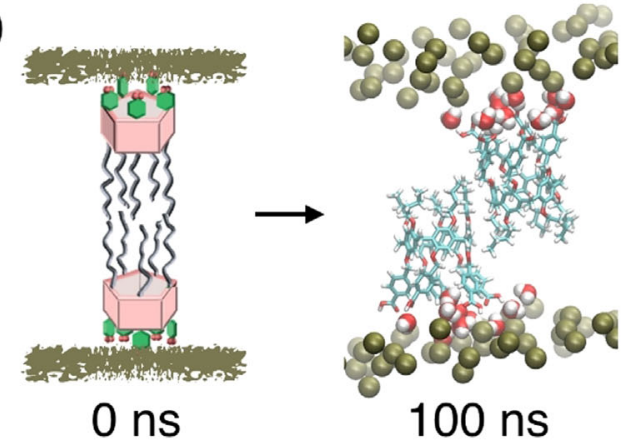

c)

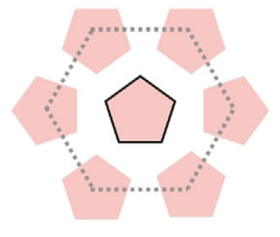

d)

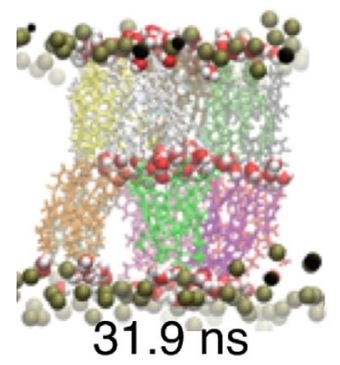

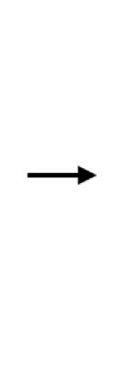

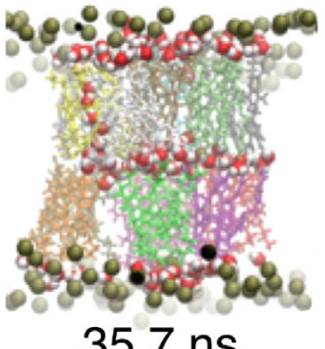

35.7 ns

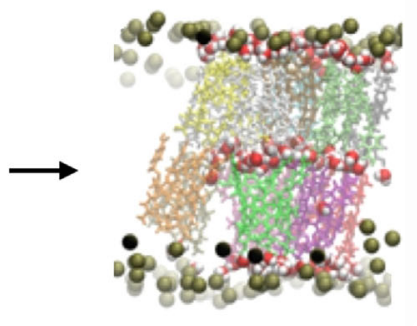

40.0 ns e)

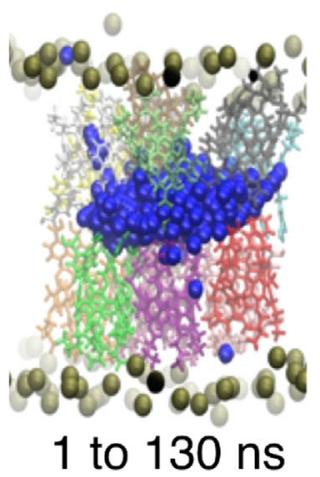

f)

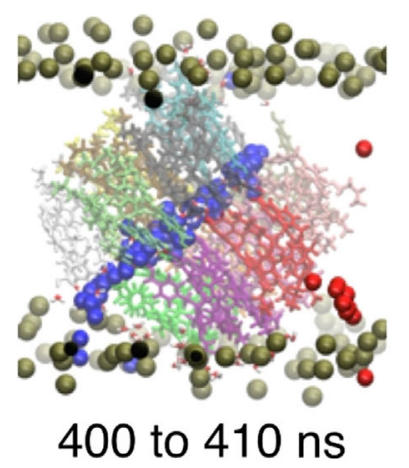

g)

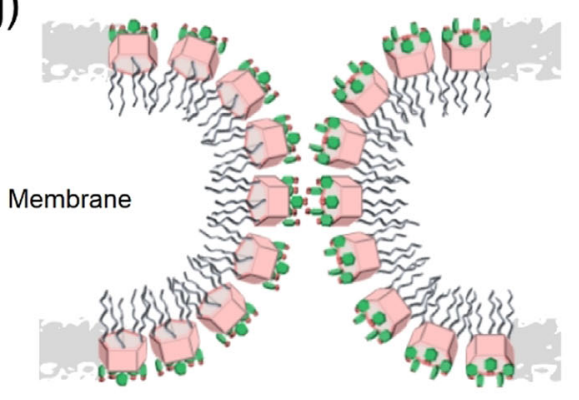

Figure 3. a) Carboxylic H-bonding dimer in a 100 ns molecular dynamics simulation in the lipid bilayer. b) Lipophilic bilayer dimer shifts side-toside. c) Top view of honeycomb hexametric arrangement around a central dimer. d) Wetting-dewetting events in the first 50 ns of the carboxylic dimer. e) Water-wire pathway in the beginning $1 \mathrm{~ns}$ after releasing constraints on the system. through the carboxyl dimer channel (around 130 ns) in the central region and the exit in a lateral exposed lipid area. $f$ ) Two water pathways are shown around 400 ns after equilibration. The blue pathway accounts for a permeation event through the toroidal $\mathrm{COOH}$ pore (during around 10 ns) and the red pathway accounts for a permeation event in the lipid area. g) Ideal schematic representation of a toroidal pore in the bilayer membrane.

we inserted a single H-bonding carboxylate or hydrophobic butyl dimers. Neither isolated dimer was stable in the membrane, leading either to a tilted but stable membraneembedded structure for carboxylate (Figure $3 \mathrm{a}$ ) or to a shifted side-by-side aggregated for butyl dimer losing its butyl connections (Figure $3 \mathrm{~b}$ ). In the latter case we hypothesize the competition with the lipid molecules to weaken the hydrophobic interactions between the butyls. Next, we tested whether larger assemblies provide increased stabilization within the membrane environment, which was indeed the case. For this purpose, we built a honeycomb arrangement where a central dimer is surrounded by a hexagon of 6 others, as schematically depicted in Figure $3 \mathrm{c}$. Both dimer assemblies form water pathways through the bilayer. At the beginning of the simulation, the carboxylate dimer honeycomb shows water wetting-dewetting forming water wires through the tubular dimers as selective pores (Figure 1d). Over time, a significant shift of the upper vs. lower layer of monomers occurs, which brings water penetration and accumulation to a halt in the region of where the carboxylic groups are present. The carboxylate dimer honeycomb does experience a tilt that is not quite as pronounced as the one observed for the monomer, but most importantly initiates water permeation events. Initially these water paths are through the dimeric pathways (Figure 1e), whereas later on alternative pathways, leading to the formation of a toroidal pore through the bilayer are observed as well (Figure $1 \mathrm{f}$ ). The permeation rate is at its maximum around $250 \mathrm{~ns}$, when the tilted structure allows a continuous water pathway around carboxylate groups. The permeation time duration varies from $2 \mathrm{~ns}$ to $175 \mathrm{~ns}$. 
Artificial toroidal pores have been pionieered by Colombini ${ }^{[34 a, b]}$ for the pores formed by natural ceramide and by our group $^{[34 c]}$ for the pores formed by artificial self-assembled crown-ethers. The inner walls of the pore would be lined with carboxylate groups and with the external butyl tails projecting into the lipid hydrocarbon region, maximizing favorable interactions with the environment for both polar and lipophilic moieties. The relatively large number of the polar carboxyl groups group relative to the hydrocarbon tail would stabilize the positive curvature required on the inside of the torus (Figure $1 \mathrm{~b}$ ). Based on their physico-chemical properties, one may hypothesize that a toroidal pore would stabilize clusters of water which probably is favored by more hydrophilic ones as proved by increased permeation rates of PADLi or PADNa. The honeycomb assembly we probed is likely not the optimal. Longer and different simulations setups will be required to investigate novel toroidal complex assemblies and are beyond the scope of the present contribution.

The present results show that, using a rationally designed pillar[5]arene platform and a simple restriction mechanism, it becomes possible to control the selectivity of water translocation in a manner reminiscent of that of proteins capable of selectively transport $\AA$ scale species. This is a very intriguing system. It is very tempting to equate the two types of structures in the solid state (hydrogen bonded and hydrophobic dimers) and the two types of channels in the bilayer membrane (dimeric H-bonded PA and a discrete large toroidal pore). The unifying assumption is that an H-bonding dimer would form preferentially and is stable in the membrane. Stacks of these dimers would lead to the dominant toroidal structure in a membrane environment providing hybrid pathways for water translocation through the dimeric water-wires and on alternative pathways encapsulating selfprotective water clusters through a toroidal pore.

I -quartet-embedded water sponges were the first example in literature taking into account the formation of $3 \mathrm{D}$ water sponges/clusters displaying high water/ion selectivity. ${ }^{[35]}$ They make the intrinsic features of water sponges ${ }^{[35]}$ or clusters $^{[3 \mathrm{~d}]}$ particularly self-protective against ions. The water translocation events lead to the change in shape of the channel conduits in bilayer membranes from a single water-wires to fully aggregated toroidal water clusters, which confer to these channels the self-adaptive behaviours.

The PAD channels showed no transport of $\mathrm{Na}^{+}$and $\mathrm{K}^{+}$ cations, making them a viable option for desalination. The observed ionic-exclusion of these channels suggests that the channel-selectivity performance is of outmost importance in translating molecular transport properties to performant membranes optimized by using chemical engineering approaches. PAD channels described here, hold significant promise for the incipient development of the innovative materials based on artificial synthetic scaffolds mimicking the functions of natural water channels. On the other hand, precise-triggered shape changes of aggregated channels also amount to coupling and transduction between geometric water clusters and selective translocation events, a feature of much interest for supramolecular translocation of water in membranes used for desalination.

\section{Acknowledgements}

This work was supported by Agence Nationale de la Recherche ANR-18-CE06-0004-02, WATERCHANNELS, the Grant-in-Aid for Scientific Research on Innovative Areas: Soft Crystal (JP18H04510 and JP20H04670), and Kiban A (JP19H00909) from MEXT Japan, JST CREST (JPMJCR18R3), and the World Premier International Research Center Initiative (WPI), MEXT, Japan.

\section{Conflict of interest}

The authors declare no conflict of interest.

Keywords: aquaporins - artificial water channels .

bilayer membranes - pillar[5]arene - supramolecular chemistry

[1] B. Hille, Ion Channels of Excitable Membranes, $3^{\text {rd }}$ ed., Sinauer Associates, Sunderland, MA, 2001

[2] a) D. A. Doyle, J. Morais Cabral, R. A. Pfuetzner, A. Kuo, J. M. Gulbis, S. L. Cohen, B. T. Chait, R. MacKinnon, Science 1998, 280, 69-77; b) E. Tajkhorshid, P. Nollert, M. O. M. Jensen, L. J. W. Miercke, J. O'Connell, R. M. Stroud, K. Schulten, Science 2002, 296, 525-530.

[3] a) Y. Le Duc, M. Michau, A. Gilles, V. Gence, Y.-M. Legrand, A. van der Lee, S. Tingry, M. Barboiu, Angew. Chem. Int. Ed. 2011, 50,11366-11372; Angew. Chem. 2011, 123, 11568-11574; b) M. Barboiu, A. Gilles, Acc. Chem. Res. 2013, 46, 2814-2282; c) R. H. Tunuguntla, R. Y. Henley, Y. C. Yao, T. A. Pham, M. Wanunu, A. Noy, Science 2017, 357, 792-796; d) W. Song, H. Joshi, R. Chowdhury, J. S. Najem, Y. X. Shen, C. Lang, C. B. Henderson, Y. M. Tu, M. Farell, M. E. Pitz, C. D. Maranas, P. S. Cremer, R. J. Hickey, S. A. Sarles, J. L. Hou, A. Aksimentiev, M. Kumar, Nat. Nanotechnol. 2020, 15, 73 - 79; J. Shen, R. J. Ye, A. Romanies, A. Roy, F. Chen, C. L. Ren, Z. Liu, H. Q. Zeng, J. Am. Chem. Soc. 2020, 142, 10050-10058.

[4] M. Barboiu, Acc. Chem. Res. 2018, 51, 2711-2718.

[5] S.-P. Zheng, L.-B. Huang, Z. Sun, M. Barboiu, Angew. Chem. Int. Ed. 2020, https://doi.org/10.1002/anie.201915287; Angew. Chem. 2020, https://doi.org/10.1002/ange.201915287.

[6] E. Mahon, S. Garai, A. Müller, M. Barboiu, Adv. Mater. 2015, 27, $5165-5170$.

[7] T. M. Fyles, C. C. Tong, New J. Chem. 2007, 31, 655-661.

[8] M. Jung, H. Kim, K. Baek, K. Kim, Angew. Chem. Int. Ed. 2008, 47, 5755-5757; Angew. Chem. 2008, 120, 5839-5841.

[9] a) O. V. Kulikov, R. Li, G. W. Gokel, Angew. Chem. Int. Ed. 2009, 48, 375-377; Angew. Chem. 2009, 121, 381-383; S. Negin, M. M. Daschbach, O. V. Kulikov, N. Rath, G. W. Gokel, J. Am. Chem. Soc. 2011, 133, 3234-3237; b) R. Li, O. V. Kulikov, G. W. Gokel, Chem. Commun. 2009, 6092-6094; c) O. V. Kulikov, M. M. Daschbach, C. R. Yamnitz, N. Rath, G. W. Gokel, Chem. Commun. 2009, 7497-7499.

[10] R. Kawano, N. Horike, Y. Hijikata, M. Kondo, A. CarnéSánchez, P. Larpent, S. Ikemura, T. Osaki, K. Kamiya, S. Kitagawa, S. Takeuchi, S. Furukawa, Chem 2017, 2, 393-403.

[11] a) T. Ogoshi, S. Kanai, S. Fujinami, T.-A. Yamagishi, Y. Nakamoto, J. Am. Chem. Soc. 2008, 130, 5022-5023; b) T. Ogoshi, T. Aoki, K. Kitajima, S. Fujinami, T.-A. Yamagishi, Y. Nakamoto, J. Org. Chem. 2011, 76, 328-331.

[12] T. Ogoshi, T. Kakuta, T. A. Yamagishi, Angew. Chem. Int. Ed. 2019, 58, 2197-2206; Angew. Chem. 2019, 131, 2219-2229.

[13] W. Si, P. Xin, Z. T. Li, J.-L. Hou, Acc. Chem. Res. 2015, 48, $1612-$ 1619. 
[14] W. Si, L. Chen, X.-B. Hu, G. Tang, Z. Chen, J.-L. Hou, Z.-T. Li, Angew. Chem. Int. Ed. 2011, 50, 12564; Angew. Chem. 2011, 123 12772.

[15] X. B. Hu, Z. Chen, G. Tang, J.-L. Hou, Z. T. Li, J. Am. Chem. Soc. 2012, 134, 8384-8387.

[16] L. Chen, W. Si, L. Zhang, G. Tang, Z. T. Li, J.-L. Hou, J. Am. Chem. Soc. 2013, 135, 2152-2155.

[17] W. Si, Z.-T. Li, J.-L. Hou, Angew. Chem. Int. Ed. 2014, 53, 4578 4581; Angew. Chem. 2014, 126, 4666-4669.

[18] Y. Zhou, Y. Chen, P. P. Zhu, W. Si, J.-L. Hou, Y. Liu, Chem. Commun. 2017, 53, 3681-3684.

[19] J.-Y. Chen, J.-L. Hou, Org. Chem. Front. 2018, 5, 1728-1736.

[20] a) M. Zhang, P.-P. Zhu, P. Xin, W. Si, Z.-T. Li, J.-L. Hou, Angew. Chem. Int. Ed. 2017, 56, 2999-3003; Angew. Chem. 2017, 129 , 3045-3049; b) W.-W. Haoyang, M. Zhang, J.-L. Hou, Chin. J. Chem. 2019, 37, 25-29.

[21] W. X. Feng, Z. Sun, M. Barboiu, Isr. J. Chem. 2018, 58, 12091218.

[22] H. Behera, L. Yang, J. L. Hou, Chin. J. Chem. 2020, 38, 215 - 217.

[23] W.-X. Feng, Z.-H. Sun, Y. Zhang, Y.-M. Legrand, E. Petit, C.-Y Su, M. Barboiu, Org. Lett. 2017, 19, 1438-1441.

[24] P. Xin, H. Kong, Y. Sun, L. Zhao, H. Fang, H. Zhu, T. Jiang, J. Guo, Q. Zhang, W. Dong, Angew. Chem. Int. Ed. 2019, 58, 27792784; Angew. Chem. 2019, 131, 2805-2810.

[25] Y.-X. Shen, W. Si, M. Erbakan, K. Decker, R. De Zorzi, P. O. Saboe, Y. J. Kang, S. Majd, P. J. Butler, T. Walz, A. Aksimentiev, J.-L. Hou, M. Kumar, Proc. Natl. Acad. Sci. USA 2015, 112 9810-9815.

[26] W. Song, H. Joshi, R. Chowdhury, J. S. Najem, Y. X. Shen, C. Lang, C. B. Henderson, Y. M. Tu, M. Farell, M. E. Pitz, C. D. Maranas, P. S. Cremer, R. J. Hickey, S. A. Sarles, J.-L. Hou, A. Aksimentiev, M. Kumar, Nat. Nanotechnol. 2020, 15, $73-79$.
[27] a) E. Licsandru, I. Kocsis, Y.-x. Shen, S. Murail, Y.-M. Legrand, A. van der Lee, D. Tsai, M. Baaden, M. Kumar, M. Barboiu, $J$. Am. Chem. Soc. 2016, 138, 5403-5409; b) M. Barboiu, Chem. Commun. 2016, 52, 5657-5665.

[28] a) D. Kaizerman-Kane, M. Hadar, N. Tal, R. Dobrovetsky, Y. Zafrani, Y. Cohen, Angew. Chem. Int. Ed. 2019, 58, 5302-5306; Angew. Chem. 2019, 131, 5356-5360; b) M. Hadar, D. Kaizerman-Kane, Y. Zafrani, Y. Cohen, Chem. Eur. J. 2020, https://doi. org/10.1002/chem.202000972.

[29] S. Fa, Y. Sakata, S. Akine, T. Ogoshi, Angew. Chem. Int. Ed. 2020, 59, 9309-9313; Angew. Chem. 2020, 132, 9395-9399.

[30] O. Mogck, V. Böhmer, W. Vogt, Tetrahedron 1996, 52, 84898496.

[31] M. L. Zeidel, S. V. Ambudkar, B. L. Smith, P. Agre, Biochemistry 1992, 31, 7436-7440.

[32] G. Yu, M. Xue, Z. Zhang, J. Li, C. Han, F. Huang, J. Am. Chem. Soc. 2012, 134, 13248-13251.

[33] S. Matile, N. Sakai in Analytical Methods in Supramolecular Chemistry (Ed.: C. A. Schalley), Wiley-VCH, Weinheim, 2007, pp. $381-418$.

[34] a) L. J. Siskind, A. Davvoody, N. Lewin, S. Marshall, M. Colombini, Biophys. J. 2003, 85, 1560-1575; b) L. Yang, T. A. Haroon, T. M. Weiss, L. Ding, H. W. Huang, Biophys. J. 2001, 81, 1475-1485; c) A. Cazacu, C. Tong, A. van der Lee, T. M. Fyles, M. Barboiu, J. Am. Chem. Soc. 2006, 128, $9541-9548$.

[35] S. Murail, T. Vasiliu, A. Neamtu, M. Barboiu, F. Sterpone, M. Baaden, Faraday Discuss. 2018, 209, 125-148.

Manuscript received: July 3, 2020

Revised manuscript received: August 17, 2020

Accepted manuscript online: September 9, 2020

Version of record online:

These are not the final page numbers! 


\section{Research Articles}

Supramolecular Chemistry

D. Strilets, S. Fa, A. Hardiagon,

M. Baaden, T. Ogoshi,*

M. Barboiu*

\section{IIII-III}

Biomimetic Approach for Highly Selective

Artificial Water Channels Based on

Tubular Pillar[5]arene Dimers

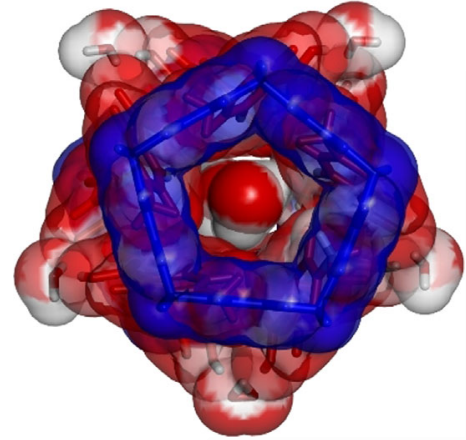

Pillar[5]arene dimers of tubular shape with variable diameters get spontaneously inserted into lipid bilayers, showing selective water conductance activity. The narrow pore gates of $\approx 2.8 \AA$, acting as selectivity filters, are perfectly sized for the water molecules to pass having themselves a diameter of $\approx 2.8 \AA$, but are restrictive enough to block the passage of hydrated $\mathrm{Na}^{+}$or $\mathrm{K}^{+}$cations.
Supramolecular Chemistry

D. Strilets, S. Fa, A. Hardiagon,

M. Baaden, T. Ogoshi,*

M. Barboiu*

\section{III-III}

Biomimetic Approach for Highly Selective

Artificial Water Channels Based on

Tubular Pillar[5]arene Dimers

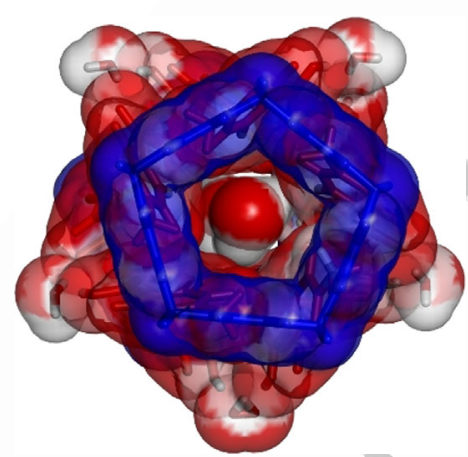

Pillar[5]arene dimers of tubular shape with variable diameters get spontaneously inserted into lipid bilayers, showing selective water conductance activity. The narrow pore gates of $\approx 2.8 \AA$, acting as selectivity filters, are perfectly sized for the water molecules to pass having themselves a diameter of $\approx 2.8 \AA$, but are restrictive enough to block the passage of hydrated $\mathrm{Na}^{+}$or $\mathrm{K}^{+}$cations.

Share your work on social media! Angewandte Chemie has added Twitter as a means to promote your article. Twitter is an online microblogging service that enables its users to send and read short messages and media, known as tweets. Please check the pre-written tweet in the galley proofs for accuracy. If you, your team, or institution have a Twitter account, please include its handle @username. Please use hashtags only for the most important keywords, such as \#catalysis, \#nanoparticles, or \#proteindesign. The ToC picture and a link to your article will be added automatically, so the tweet text must not exceed 250 characters. This tweet will be posted on the journal's Twitter account (follow us @angew_chem) upon publication of your article in its final (possibly unpaginated) form. We recommend you to re-tweet it to alert more researchers about your publication, or to point it out to your institution's social media team.

Please check that the ORCID identifiers listed below are correct. We encourage all authors to provide an ORCID identifier for each coauthor. ORCID is a registry that provides researchers with a unique digital identifier. Some funding agencies recommend or even require the inclusion of ORCID IDs in all published articles, and authors should consult their funding agency guidelines for details. Registration is easy and free; for further information, see http://orcid.org/.

Dmytro Strilets

Dr. Shixin Fa

Arthur Hardiagon

Dr. Marc Baaden

Prof. Tomoki Ogoshi

Dr. Mihail Barboiu http://orcid.org/0000-0003-0042-9483

Author Contributions

D.S. Data curation: Supporting; Formal analysis: Supporting; Investigation: Supporting

S.F. Data curation: Supporting; Investigation: Supporting. 